\title{
ANZAHL DER BESTÄTIGTEN FÄLLE VON SYPHILIS BEI SCHWANGEREN IN BRASILIEN ZWISCHEN 2009 UND 2013
}

\section{ORIGINALER ARTIKEL}

BARROS, Yara Lorrane Souza de ${ }^{1}$, FECURY, Amanda Alves², OLIVEIRA, Euzébio $\mathrm{de}^{3}$, DENDASCK, Carla Viana ${ }^{4}$, ARAÚJO, Maria Helena Mendonça de ${ }^{5}$, SOUZA, Keulle Oliveira da ${ }^{6}$, DIAS, Claudio Alberto Gellis de Mattos ${ }^{7}$

BARROS, Yara Lorrane Souza de. Et al. Anzahl der bestätigten Fälle von Syphilis bei Schwangeren in Brasilien zwischen 2009 und 2013. Revista Científica Multidisciplinar Núcleo do Conhecimento. Jahrgang 05, Ed. 11, Vol. 25, S. 53-61. November 2020. ISSN: 2448-0959, Zugangslink: https://www.nucleodoconhecimento.com.br/gesundheit/syphilis-bei-schwangeren, DOI: 10.32749/nucleodoconhecimento.com.br/gesundheit/syphilis-bei-schwangeren

\section{ZUSAMMENFASSUNG}

Syphilis ist eine bakterielle Erkrankung, die von Schwangeren mit Syphilis auf den Fötus übertragen wird. Die Diagnose Syphilis bei Schwangeren wird durch die VDRL-Untersuchung bestätigt. Ziel dieser Arbeit ist es, die Anzahl der bestätigten Fälle von Syphilis bei Schwangeren in Brasilien als Jahr der Diagnose, Altersgruppe, Regionen Brasiliens, Wohngebiet, Art der Prüfung, Klassifizierung und Evolution zwischen 2009 und 2013 zu zeigen. Sekundärdaten wurden von der Edv-Abteilung

\footnotetext{
${ }^{1}$ Bergbautechnik, als Ergebnis des Bundesinstituts für Amapá (IFAP).

${ }^{2}$ Biomedizin, PhD in Tropenkrankheiten, Professor und Forscher des Medizinischen Studiengangs der Föderalen Universität Amapá (UNIFAP).

${ }^{3}$ Biologe, PhD in Topische Krankheiten, Professor und Forscher des Leibeserziehungskurses der Föderalen Universität Pará (UFPA).

${ }^{4}$ Theologe, PhD in Psychoanalyse, Forscher am Zentrum für Forschung und Höhere Studien - CEPA.

${ }^{5}$ Arzt, Professor und Forscher des Medizinischen Studiengangs der Föderalen Universität Amapá (UNIFAP).

${ }^{6}$ Soziologe, Master-Student in Anthropischen Studien im Amazonas, Mitglied der Forschungsgruppe "Labor für Bildung, Umwelt und Gesundheit" (LEMAS/UFPA).

${ }^{7}$ Biologe, PhD in Theorie- und Verhaltensforschung, Professor und Forscher des Graduiertenprogramms für berufliche und technologische Bildung (PROFEPT), Bundesinstitut für Amapá (IFAP).
}

RC: 68389

Verfügbar in: https://www.nucleodoconhecimento.com.br/gesundheit/syphilis-bei- 
von SUS - DATASUS (http://datasus.saude.gov.br) verwendet. Die größere Anzahl von Tests ermöglicht eine bessere Behandlung und Verringerung der Anzahl der Fälle. Das Fehlen einer vorbeugenden Betreuung während der sexuellen Beziehung führt jedoch zu einer Zunahme der Fälle, insbesondere bei Frauen im reproduktiven Alter. Gebiete mit einer größeren Bevölkerung weisen eine höhere Zahl von Fällen auf. Die hohe Variation der Partner in diesen Bereichen führt zu einer erhöhten Kontamination. Die Art des Tests beeinflusst die Zahlen. Weniger spezifische, allgemeinere Tests finden spezifische und unspezifische Antikörper für das Bakterium. Dadurch können die tatsächlichen Zahlen geändert werden. Die weibliche Gesundheitsversorgung macht sowohl die Diagnose früh, erleichtert die Behandlung. Syphilis ist nicht direkt verantwortlich für die höhere Sterblichkeit bei Schwangeren.

Stichworte: Epidemiologie, Syphilis, Schwangere.

\section{EINFÜHRUNG}

Syphilis ist eine bakterielle Erkrankung, die den ganzen Körper betrifft, sexuell übertragen; durch Blutspende; direkter Kontakt mit kontaminiertem Blut; und die schwangere Frau mit Syphilis für den Fötus. Das krankheitserregende Bakterium ist Treponema Pallidum (CARVALHO et al., 2014, JUNIOR et al., 2009; ROCHA et al., 2020).

Symptome der Syphilis manifestieren sich in drei Phasen. In der ersten Phase können rote Flecken am Körper erscheinen, Wunden in der Regel an den Genitalien. In der zweiten Phase ist es möglich, dass die Krankheit für einen Zeitraum von bis zu acht Monaten schlummert, wenn das Bakterium verteilt werden kann, das die Haut und Organe beeinflusst. In der dritten Phase tritt es häufig zu Entzündungen im Gewebe des Körpers, wie Knochen, Muskeln und Leber (AVELLEIRA et al., 2006; BRETAS et al., 2008).

RC: 68389

Verfügbar in: https://www.nucleodoconhecimento.com.br/gesundheit/syphilis-bei- 
Die Krankheit muss diagnostiziert werden, damit der Patient die entsprechende Behandlung durch die Verwendung von männlichen/weiblichen Kondomen verhindern kann. Die wirksamste Behandlung ist das Medikament Benzaycin Penicillin. Bis 2015 gab es keine Aufzeichnungen über Fälle von Widerstand gegen sie. Andere Medikamente werden bei der Behandlung von Syphilis verwendet, aber dasjenige, das die höchste Effizienz in der Behandlung/Heilung hat, ist diese Art von Penicillin (KALININ et al., 2015; BRASIL, 2015).

Die Diagnose der Syphilis bei Schwangeren wird durch die VDRL (Venereal Disease Research Laboratory) Untersuchung während der pränatalen Versorgung bestätigt. Die Entdeckung der Krankheit und die Behandlung von Schwangeren während der pränatalen Behandlung mit Penicillin verhindern angeborene Syphilis (SUTO et al., 2016; MESQUITA et al., 2012).

Die Zahl der Syphilisfälle bei Schwangeren in Brasilien lag 2012 und 2013 bei 21382 (BRASIL 2015).

Die Zahl der Syphilisfälle bei Schwangeren im Nordosten lag 2012 und 2013 bei 4.433. Im gleichen Zeitraum betrug die Zahl der Fälle von Syphilis bei Schwangeren in der Region Südosten 10.052; in der Region Süd waren 2795; in der Region mittlerer Westen war 1961; und in der Region Nord waren es 214 (BRASIL, 2015).

Die Zahl der Syphilis-Fälle bei Schwangeren in Amapa betrug 176 in den Jahren 2012 und 2013 (BRASIL 2015).

\section{TOR}

Ziel dieses Artikels ist es, die Anzahl der bestätigten Fälle von Syphilis bei Schwangeren in Brasilien zu zeigen, wie das Diagnosejahr, Altersgruppe, Regionen Brasiliens, Wohngebiet, Art des Tests, Klassifizierung und Evolution zwischen 2009 und 2013.

RC: 68389

Verfügbar in: https://www.nucleodoconhecimento.com.br/gesundheit/syphilis-bei- 


\section{SYSTEM}

Daten aus der Edv-Abteilung von SUS - DATASUS (http://datasus.saude.gov.br) nach folgenden Schritten: Zuerst wurde die Registerkarte "Zugriff auf Informationen" ausgewählt, gefolgt von der Option "Gesundheitsinformationen (TABNET)" kurz nach der Option "Epidemiologie und Morbidität". Es klickte auf die Option "Krankheiten und Krankheiten der Berichterstattung ab 2007 (SINAN)" und dann "Syphilis in Schwangeren", und in der Registerkarte "geografische Abdeckung" ausgewählt "Brasilien nach Region, UF und Gemeinde". Die nächsten Schritte waren: A) in der ausgewählten Zeile "Bestätigte Fälle nach Diagnosejahr", in der Spalte "nicht aktiv" und inhaltlich "bestätigte Fälle". Für diese Option und für die folgenden Daten wurden von 2009 bis 2013 gesammelt. B) in der Zeile wurden in der Spalte "Nicht-aktiv" und in "bestätigten Fällen" "bestätigte Fälle" ausgewählt. C) in der Zeile wurden "Bestätigte Fälle nach Altersgruppe" in der Spalte "nicht aktiv" und in "bestätigten Fällen" ausgewählt. D) in der Zeile "Fälle, die nach Evolution bestätigt wurden", wurden in der Spalte "nicht aktiv" und inhaltlich "bestätigte Fälle" ausgewählt. E) in der Zeile "Bestätigte Fälle nach klinischer Klassifikation", in der Spalte "nicht aktiv" und inhaltlich "bestätigte Fälle" ausgewählt. F) in der Zeile "Bestätigte Fälle nach Nicht-Trep-Test", in der Spalte "nicht aktiv" und inhaltlich "bestätigte Fälle" ausgewählt. G) in der Zeile wurden "Bestätigte Fälle nach Trep-Test" in der Spalte "nicht aktiv" in "bestätigten Fällen" ausgewählt. H) wählen Sie in der Zeile "Bestätigte Fälle nach Zone Residence zweites Diagnosejahr", in der Spalte "Wohnzone" und im Inhalt "bestätigte Fälle". Die Daten wurden in der Excel-Anwendung, einer Komponente der Microsoft Corporation Office-Suite, kompiliert. Die bibliographische Forschung wurde in wissenschaftlichen Artikeln mit Computern aus dem Computerlabor des Bundesinstituts für Bildung, Wissenschaft und Technologie von Amap, Macapa Campus, unter: Rodovia BR 210 KM 3, s/n - Bairro Brasil Novo durchgeführt. CEP: 68.909-398, Macapá, Amapá, Brasil.

$\mathrm{RC}: 68389$

Verfügbar in: https://www.nucleodoconhecimento.com.br/gesundheit/syphilis-bei- 


\section{ERGEBNISSE}

Abbildung 1 zeigt die Zahl der bestätigten Fälle von Syphilis bei Schwangeren pro Jahr in Brasilien zwischen 2009 und 2013. Die Daten zeigen einen Anstieg der Zahl der bestätigten Fälle von 2009 bis 2012. Diese Zahl ging 2013 im Vergleich zum Vorjahr um die Hälfte zurück.

Abbildung 1 Anzahl der bestätigten Fälle von Syphilis bei Schwangeren pro Jahr in Brasilien zwischen 2009 und 2013.

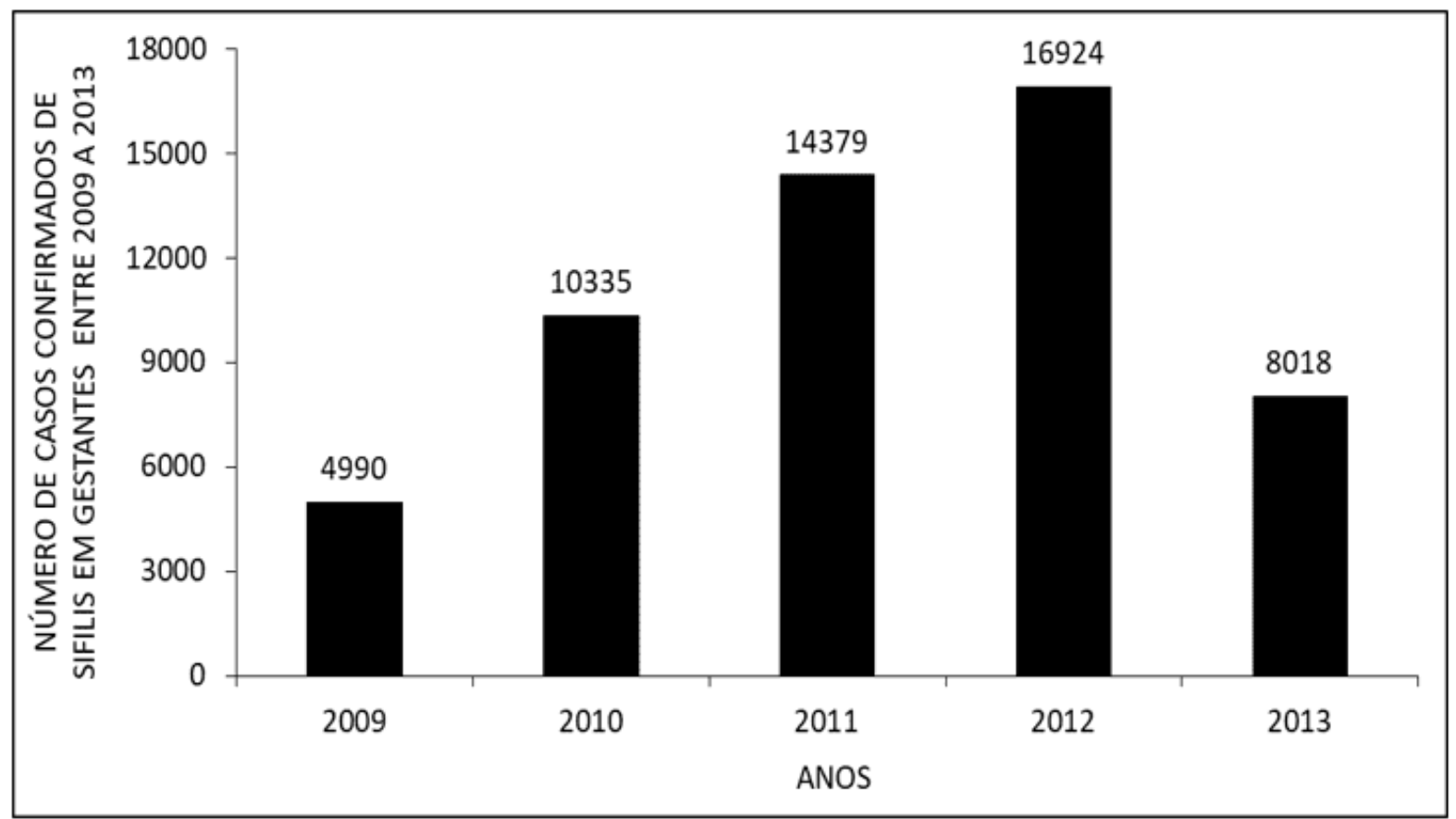

Abbildung 2 zeigt die Anzahl der bestätigten Fälle von Syphilis bei Schwangeren nach Altersgruppen in Brasilien zwischen 2009 und 2013. Zwischen 10 und 39 Jahren gab es ein Wachstum. Diese Zahl sinkt zwischen 40 und 64 Jahren.

$\mathrm{RC}: 68389$

Verfügbar in: https://www.nucleodoconhecimento.com.br/gesundheit/syphilis-bei- 
Abbildung 2 Anzahl der bestätigten Fälle von Syphilis bei Schwangeren nach Altersgruppen in Brasilien zwischen 2009 und 2013.

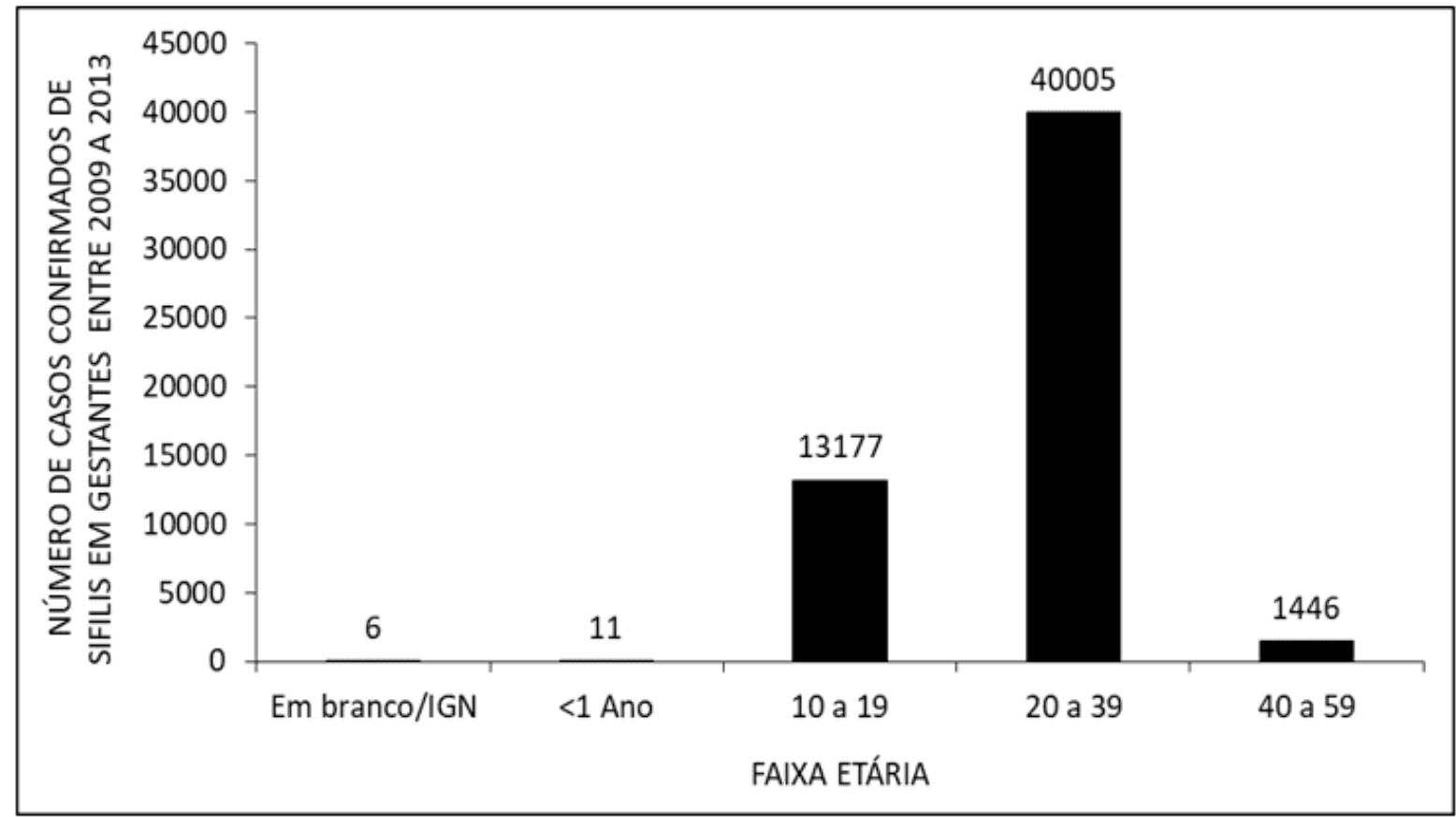

Abbildung 3 zeigt die Anzahl der bestätigten Fälle von Syphilis bei Schwangeren nach Regionen in Brasilien zwischen 2009 und 2013. Die südöstliche Region weist die höchste Anzahl bestätigter Fälle auf, gefolgt von den Regionen Im Nordosten, Norden, Süden und Mittleren Westen.

$\mathrm{RC}: 68389$

Verfügbar in: https://www.nucleodoconhecimento.com.br/gesundheit/syphilis-bei- 
Abbildung 3 Anzahl der bestätigten Fälle von Syphilis bei Schwangeren nach Regionen des Landes in Brasilien zwischen 2009 und 2013.

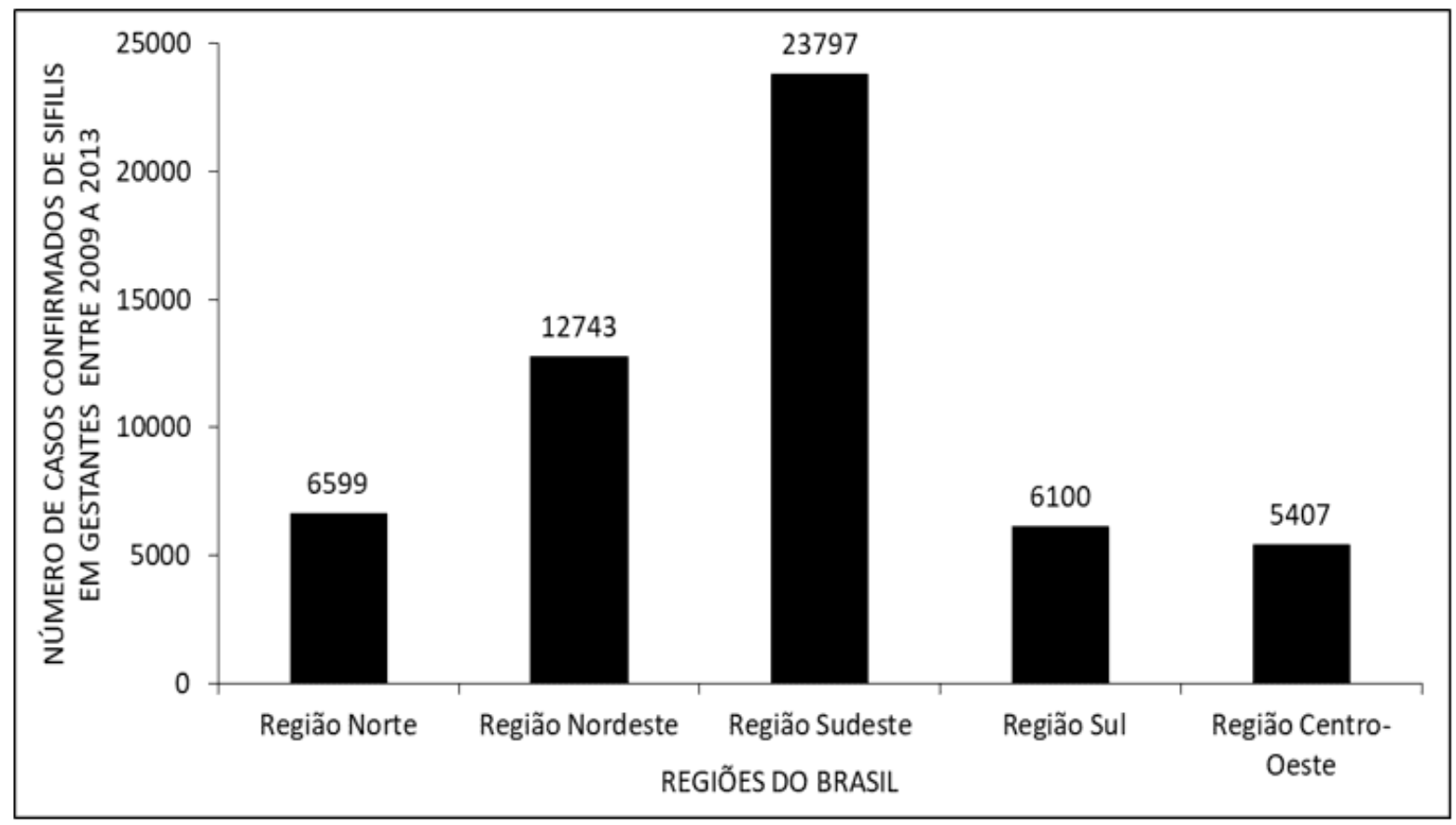

Abbildung 4 zeigt die Zahl der bestätigten Fälle von Syphilis bei Schwangeren pro Wohnsitzgebiet in Brasilien zwischen 2009 und 2013. Die Zahl im Stadtgebiet ist etwa achtmal höher als im ländlichen Raum. Die niedrigste Zahl von Fällen pro Wohngebiet ist periurban bzw. Ign/Branco.

RC: 68389

Verfügbar in: https://www.nucleodoconhecimento.com.br/gesundheit/syphilis-bei- 
Abbildung 4 Anzahl der bestätigten Fälle von Syphilis bei Schwangeren nach art des Wohnsitzgebietes in Brasilien zwischen 2009 und 2013.

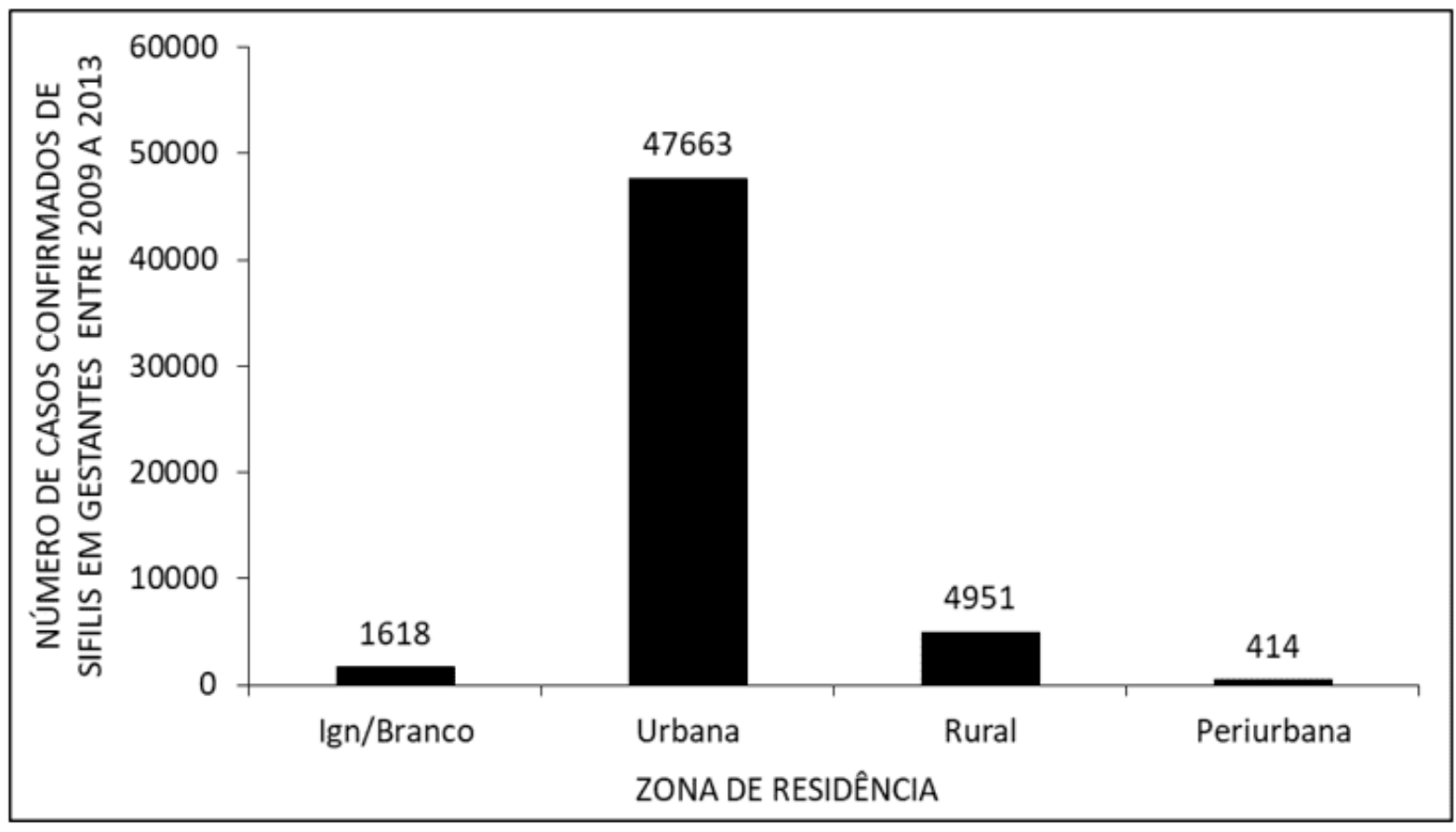

Abbildung 5 zeigt die Anzahl der bestätigten Fälle von Syphilis bei Schwangeren nach Art des Tests, der zwischen 2009 und 2013 in Brasilien durchgeführt wurde. Die Anzahl der Pro-TREP-Tests durchgeführten Ign/White-Tests ist um Die FrameZeiten höher als die der NON-TREP-Tests. Die Anzahl der reaktiven Fälle durch NON-TREP-Tests ist fast doppelt so viele Fälle, die durch TREP-Tests reaktiv sind. Die Anzahl der nicht reaktiven Fälle zwischen NON-TREP- und TREP-Tests ist ungefähr. Die Anzahl der nicht durchgeführten Fälle von TREP-Tests ist neunmal höher als die Anzahl der Fälle, die nicht von NON-TREP-Tests durchgeführt wurden.

RC: 68389

Verfügbar in: https://www.nucleodoconhecimento.com.br/gesundheit/syphilis-bei- 
Abbildung 5 Anzahl der bestätigten Fälle von Syphilis bei Schwangeren nach der Art des Tests, der zwischen 2009 und 2013 in Brasilien durchgeführt wurde.

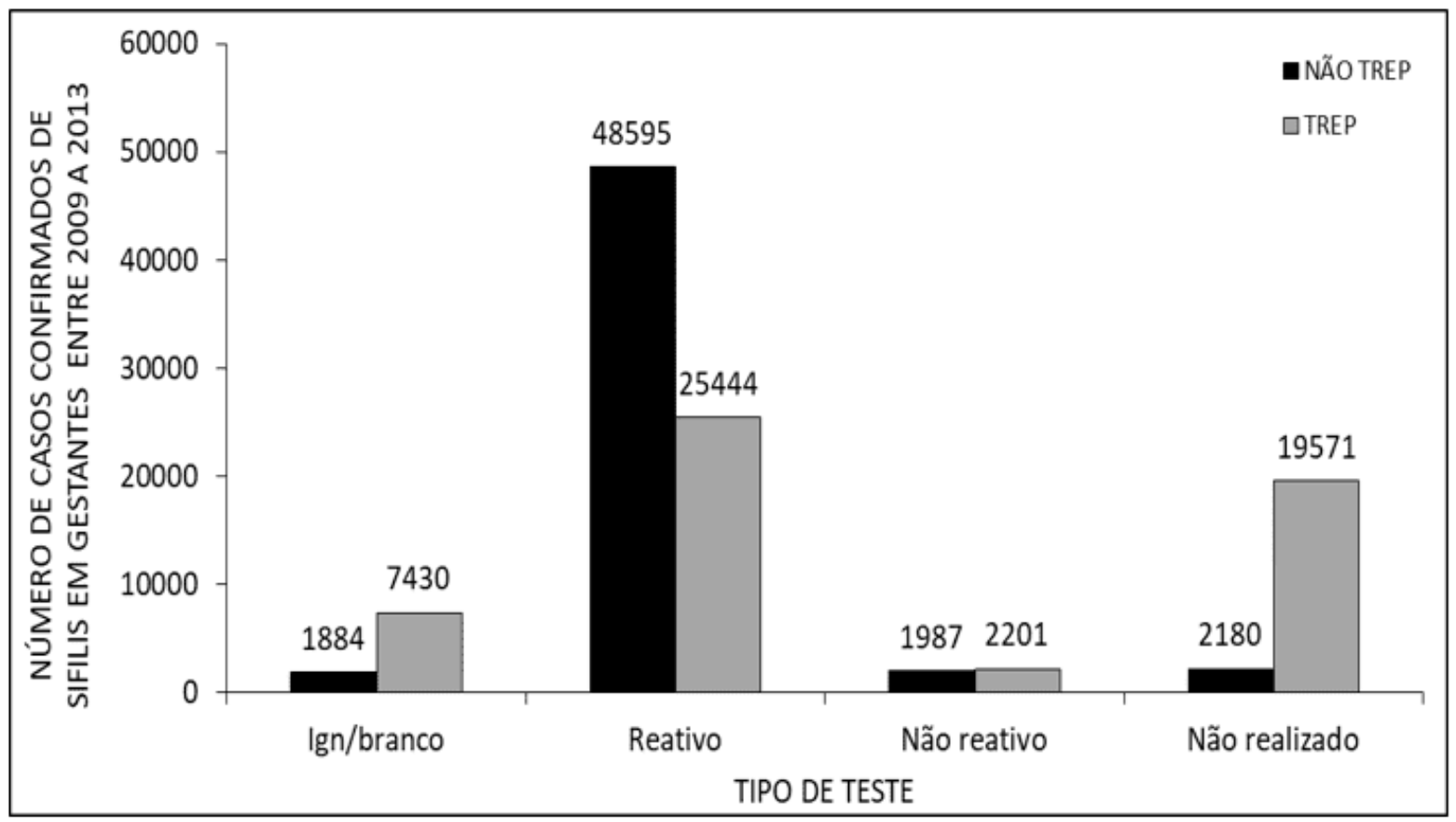

Abbildung 6 zeigt die Anzahl der bestätigten Fälle von Syphilis bei Schwangeren gemäß der Klassifikation in Brasilien zwischen 2009 und 2013. Die primäre Klassifizierung hat die höchste Anzahl von Fällen, gefolgt von Ign/White und latent. Die Klassifikationen, sekundäre und tertiäre, weisen die niedrigste Anzahl bestätigter Fälle auf.

RC: 68389

Verfügbar in: https://www.nucleodoconhecimento.com.br/gesundheit/syphilis-bei- 
Abbildung 6 Anzahl der bestätigten Fälle von Syphilis bei Schwangeren gemäß der Klassifikation in Brasilien zwischen 2009 und 2013.

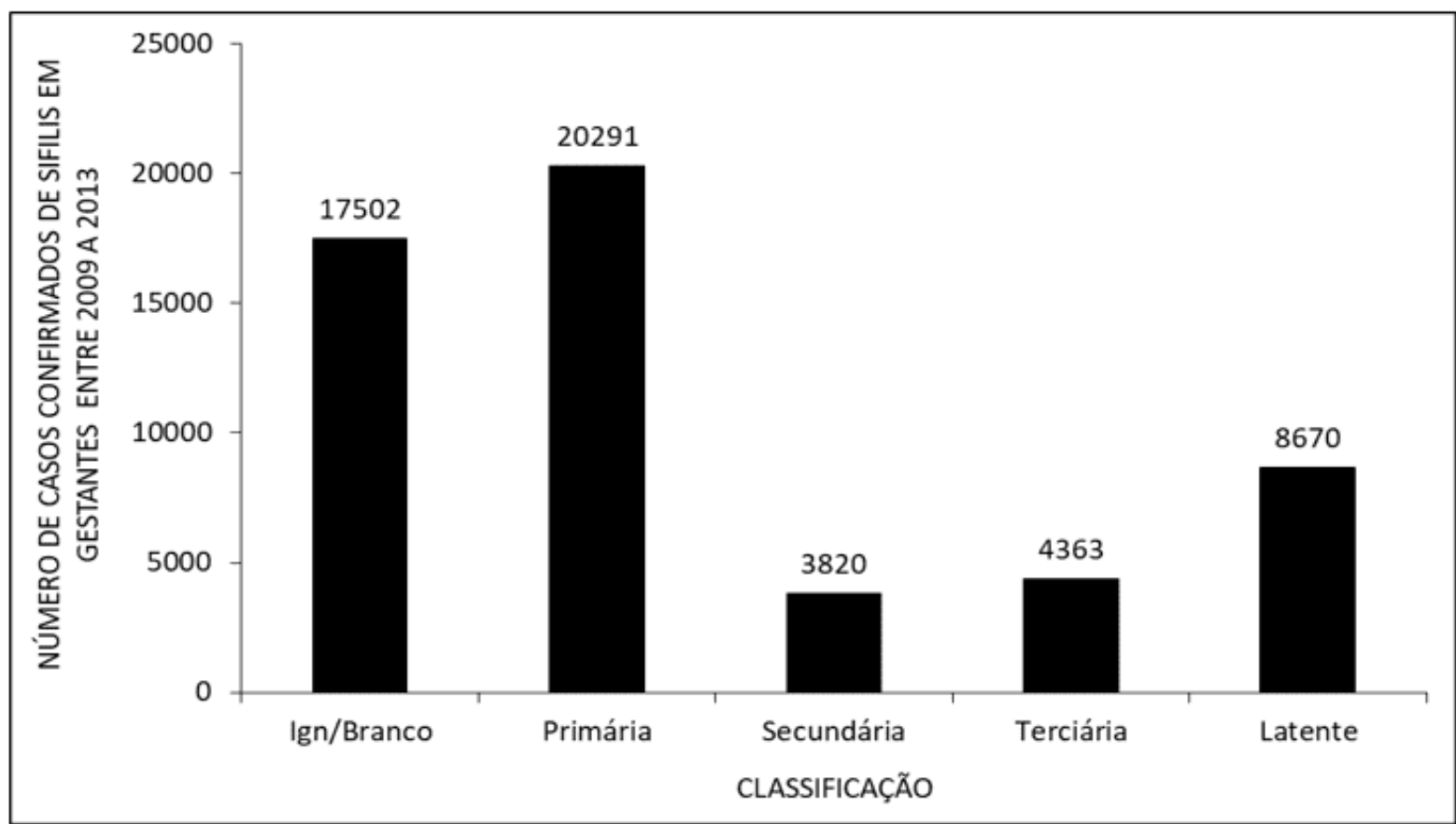

Abbildung 7 zeigt die Anzahl der bestätigten Fälle von Syphilis bei Schwangeren gemäß der Entwicklung in Brasilien zwischen 2009 und 2013. Die Daten zeigen, dass die Zahl der Todesfälle von Schwangeren, die Syphilis in diesem Zeitraum erworben haben, doppelt so viele wie die Zahl der Heilungen war. Die höchste Zahl von Todesfällen gab es aufgrund anderer Ursachen. Die niedrigste Zahl von Todesfällen in diesem Zeitraum wurde als untersucht angezeigt.

RC: 68389

Verfügbar in: https://www.nucleodoconhecimento.com.br/gesundheit/syphilis-bei- 
Abbildung 7 Anzahl der bestätigten Fälle von Syphilis bei Schwangeren nach der Entwicklung in Brasilien zwischen 2009 und 2013.

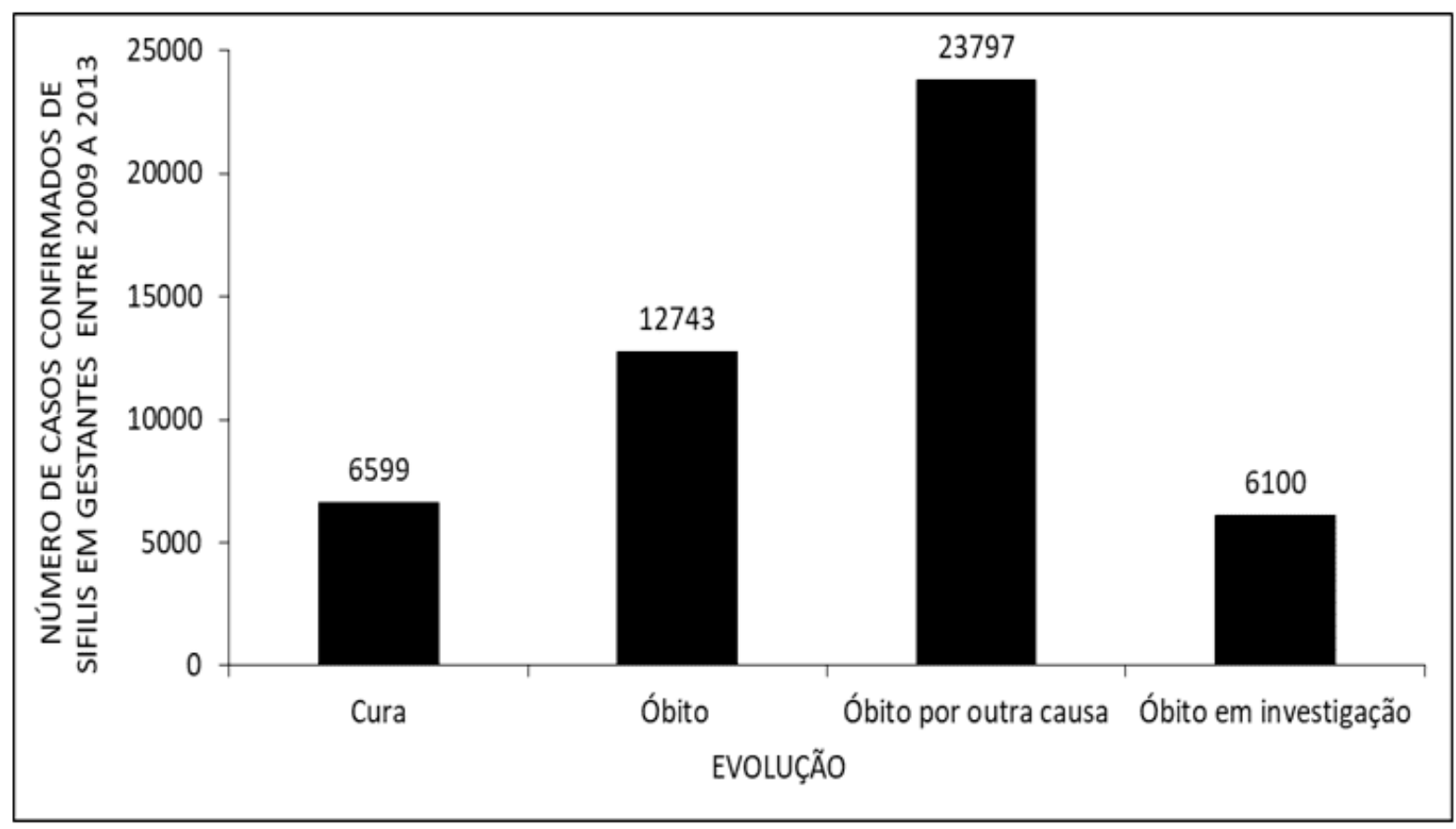

\section{DISKUSSION}

Zwischen 2009 und 2012 stieg die Zahl der bestätigten Fälle von Syphilis bei Schwangeren. Zwischen 2011 und 2013 stieg die Zahl der Tests von 31,5000 auf 1,7 Millionen, was es möglich zu haben scheint, den Patienten mit Syphilis zu behandeln. Dieses Szenario könnte den Rückgang der bestätigten Fälle bei Schwangeren im Jahr 2013 beeinflusst haben (BRASIL, 2013; 2017).

Die Daten zeigen Fälle von Syphilis bei Schwangeren zwischen 10 und 39 Jahren und ihr Rückgang zwischen 40 und 64 Jahren (Abbildung 2), bestätigt durch die Literatur. In der Altersgruppe zwischen 20 und 34 Jahren befinden sich Frauen auf dem Höhepunkt ihrer Fortpflanzungsphase. Es scheint eine höhere Rate von Schwangeren mit Syphilis in dieser Altersgruppe zu geben, möglicherweise erklärt durch die Praxis des ungeschützten Geschlechts (COSTA et al, 2012).

RC: 68389

Verfügbar in: https://www.nucleodoconhecimento.com.br/gesundheit/syphilis-bei- 
Der Südosten ist die brasilianische Region mit der höchsten Anzahl von SyphilisFällen bei Schwangeren im untersuchten Zeitraum. Diese Daten lassen sich damit erklären, dass der Südosten die Mehrheit der brasilianischen Bevölkerung konzentriert und etwa die Hälfte der Bevölkerung zugibt, keine Kondome in sexuellen Beziehungen zu verwenden (BRASIL, 2011; IBGE, 2010).

Im untersuchten Zeitraum stellte das Stadtgebiet Brasiliens eine höhere Zahl von Fällen von Schwangeren mit Syphilis als im ländlichen Raum auf (Abbildung 4). Der Literatur zufolge sind Menschen aus der städtischen Bevölkerung sexuell mit einer größeren Anzahl von Partnern verwandt. Dies kann zu einer Erhöhung der Wahrscheinlichkeit führen, dass eine Person ohne einen stabilen oder festen Sexualpartner sexuell übertragbare Infektionen wie Syphilis erwirbt (BRASIL, 2011).

Die höchste Anzahl bestätigter Fälle war bei den NON-TREP-Tests als bei den TREP-Tests. Dies lag wahrscheinlich daran, dass TREP-Tests spezifischer sind, indem sie nur Antikörper gegen das Bakterium lokalisieren, während NON-TREPTests spezifische und unspezifische Antikörper gegen das Bakterium Treponema pallidum lokalisieren (BRASIL, 2015).

Es gab eine hohe Anzahl von bestätigten Fällen von Syphilis bei Schwangeren nach Primärklassifikation. Es wird angenommen, dass die Patientin nach dem ersten Symptom, in diesem Fall hartem Krebs, eine Krankenhausbehandlung sucht, wenn sie die Diagnose der primären Syphilis erhält (AVELLEIRA et al., 2006).

Die höchste Zahl von Todesfällen bei Schwangeren mit Syphilis war auf eine andere Ursache zurückzuführen. Der Literatur zufolge waren mehr als die Hälfte der Todesfälle von Müttern weltweit von Blutungen, Bluthochdruck und Septämie (SAY, 2014) verursacht worden.

RC: 68389

Verfügbar in: https://www.nucleodoconhecimento.com.br/gesundheit/syphilis-bei- 


\section{FAZIT}

Die größere Anzahl von Tests ermöglicht eine bessere Behandlung und Verringerung der Anzahl der Fälle. Das Fehlen einer vorbeugenden Betreuung während der sexuellen Beziehung führt jedoch zu einer Zunahme der Fälle, insbesondere bei Frauen im reproduktiven Alter.

Gebiete mit einer größeren Bevölkerung weisen eine höhere Zahl von Fällen auf. Die hohe Variation der Partner in diesen Bereichen führt zu einer erhöhten Kontamination.

Die Art des Tests beeinflusst die Zahlen. Weniger spezifische, allgemeinere Tests finden spezifische und unspezifische Antikörper für das Bakterium. Dadurch können die tatsächlichen Zahlen geändert werden. Die weibliche Gesundheitsversorgung macht sowohl die Diagnose früh, erleichtert die Behandlung.

Syphilis ist nicht direkt verantwortlich für die höhere Sterblichkeit bei Schwangeren.

\section{REFERENZEN}

AVELLEITA, J. C. R.; BOTTINO, G; Sífilis: Diagnóstico, Tratamento e Controle. An. Bras. Dermatol, Vol.81 no.2 Rio de Janeiro Mar./Apr. 2006.

BRASIL São Paulo, Centro de Referência e Treinamento DST/Aids. Boletim Epidemiológico. Ano XXX. 2013.

BRASIL, Ministério da Saúde. Secretaria de Ciência, Tecnologia e Insumos Estratégicos. Relatório de Recomendação, n¹59, 2015. Disponível em: <file:///C:/Users/aluno.SELABI/Desktop/CURSO\%20DE\%20ESCRITA/(BRASIL,\%20 2015).pdf>. Acesso em:26/09/2017.

RC: 68389

Verfügbar in: https://www.nucleodoconhecimento.com.br/gesundheit/syphilis-bei- 
BRASIL. Ministério da Saúde Secretaria de Vigilância em Saúde - Departamento de DST, Aids e Hepatites Virais SAF SUL Trecho 2 Boletim Epidemiológico - Sífilis Ano IV- no 1, 2015.

BRASIL. Ministério da Saúde. Ministério da Saúde Lança Campanha de Combate à Sífilis. Disponível em: <http://www.brasil.gov.br/saude/2013/10/ministerio-dasaude-lanca-campanha-de-combate-a-sifilis>. Acesso em: 19/09/2017.

BRASIL. Ministério da Saúde. Portal da Saúde. Combate á Sífilis Congênita. Disponivel em: <http://portalarquivos.saude.gov.br/campanhas/sifilis/>. Acesso em: 19/09/2017.

BRASIL. Ministério da Saúde. Secretaria de Vigilância em Saúde. Departamento de DST, Aids e Hepatites Virais. Pesquisa de conhecimento, atitudes e práticas na população brasileira / Ministério da Saúde. Secretaria de Vigilância em Saúde. Departamento de DST, Aids e Hepatites Virais. - Brasília: Ministério da Saúde, 2011.

BRASIL. Ministério da Saúde. Secretaria de Vigilância em Saúde. Departamento de DST, Aids e Hepatites Virais. Pesquisa de conhecimento, atitudes e práticas na população brasileira / Ministério da Saúde. Secretaria de Vigilância em Saúde. Departamento de DST, Aids e Hepatites Virais. - Brasília: Ministério da Saúde, 2011.

BRETAS, J. R. S.; OHARA, C. V. S.; JARDIM, D. P.; MUROYA, R. L.; Conhecimento Sobre DST/AIDS por Estudantes Adolescentes. Rev. esc. enferm. USP vol.43 no.3 São Paulo Sept. 2009.

CARVALHO, I. S.; BRITO, R. S; Sífilis congênita no Rio Grande do Norte: estudo descritivo do período 2007-2010. Epidemiol. Serv. Saúde, v.23 n.2 Brasília, 2014.

RC: 68389

Verfügbar in: https://www.nucleodoconhecimento.com.br/gesundheit/syphilis-bei- 
COSTA, C. C.; FEITAS, L. V.; SOUSA, D. M. N.; OLIVEIRA, L. L.; CHAGAS, A. C. M. A.; LOPES, M. V. O.; DAMASCENO, A. K. C. Sífilis Congênita No Ceará: Análise Epidemiológica De Uma Década. Rev Esc Enferm USP 2013; 47(1):149-56.

IBGE, Censo Demográfico 1960, 1970, 1980, 1991, 2000 e 2010. Disponível em: $<$ https://censo2010.ibge.gov.br/sinopse/index.php?dados=8>. Acesso em: 26/09/2017.

JUNIOR, W. B.; SHIRATSU, R.; PINTO, V.; Abordagem nas Doenças Sexualmente Transmissíveis. An Bras Dermatol. 2009;84(2):151-59.

KALININ, Y.; NETO, A. P.; PASSARELLI, D. H. C. Sífilis: aspectos clínicos, transmissão, Manifestações orais, diagnóstico e tratamento. Odonto 2015; 23(4546): 65-76.

MESQUITA, K.; LIMA, G. K.; FILGUEIRA, A.; FLÔR, S. M.; FREITAS, C. A.; LINHARES, M. S.; GUBERT, F.Análise dos Casos de Sífilis Congênita em Sobral, Ceará: Contribuições para Assistência Pré-Natal. DST - J bras Doenças Sex Transm 2012; 24(1):20-27

ROCHA, K.D., FECURY, A.A., OLIVEIRA, E., DENDASCK, C.V., DIAS, C.A.G.M. Number of congenital syphilis cases in Brazil between 2009 and 2013. Revista Científica Multidisciplinar Núcleo do Conhecimento. Year 05, Ed. 05, Vol. 01, pp. 131-143. May 2020.2 ISSN:2448-0959. DOI: 10.32749/nucleodoconhecimento.com.br/health/congenital-syphilis-cases.

SAY, L.; CHAU, D.; GEMMILL, A.; TUBÇALP, O.; MOLLER, A.; DANIELS, J.; GULMEZOGLU, A. M.; TEMMERMAN, M.; ALKEMA, L. Global Causes Of Maternal Death: A Who Systematic Analysis. Lancet Glob Health, Vol.2, 2014.

RC: 68389

Verfügbar in: https://www.nucleodoconhecimento.com.br/gesundheit/syphilis-bei- 
SUTO, C. S. S.; SILVA, D. L.; ALMEIDA, E. S.; COSTA, E. L.; EVANGELISTA, T. J. Assistência Pré-Natal A Gestante Com Diagnóstico De Sífilis Revista de Enfermagem e Atenção à Saúde 2016; 5(2): 18-33.

Eingereicht: November 2020.

Genehmigt: November 2020.

RC: 68389

Verfügbar in: https://www.nucleodoconhecimento.com.br/gesundheit/syphilis-bei- 